\section{ACTIVATED OSTEOARTHRITIS FOLLOWING IMMUNE CHECKPOINT INHIBITOR TREATMENT: AN OBSERVATIONAL STUDY}

${ }^{1}$ Pankti Reid*, ${ }^{2}$ David Liew, ${ }^{3}$ Rajshi Akruwala, ${ }^{4}$ Anne Bass, ${ }^{4}$ Karmela Chan. ${ }^{1}$ University of Chicago Medical Center, Chicago, IL, United States; ${ }^{2}$ University of Melbourne, Parkville VIC, Parkville VIC, Australia; ${ }^{3}$ SUNY Downstate Medical Center, New York, NY, United States; ${ }^{4}$ HospitalforSpecial Surgery, Weill Cornell, Chicago, IL, United States

Background Immune checkpoint inhibitors (ICIs) have revolutionized cancer therapy but can result in toxicities, known as immune-related adverse events (irAEs), due to a hyperactivated immune system. ICI-related inflammatory arthritis has been described in literature, but herewith we introduce and characterize post-ICI activated osteoarthritis (ICI-aOA).

Methods We conducted a multi-center, retrospective, observational study of patients with cancer treated with ICIs and diagnosed with ICI-aOA by a rheumatologist. ICI-aOA was defined by (1) an increase in non-inflammatory joint pain after ICI initiation, (2) in joints characteristically affected by osteoarthritis and (3) lack of inflammation on exam. Cases were graded using the CTCAE (Common Terminology Criteria for Adverse Events) V6.0 rubric for arthralgia. RECIST (Response evaluation criteria in solid tumors) V.1.1 (v.4.03) guidelines determined tumor response. Results were analyzed using Chi-squared tests of association and multivariate logistic regression.

Results Thirty-six patients had ICI-aOA with mean age at time of rheumatology presentation of 66 years (51-81yrs). Most patients had metastatic melanoma $(10 / 36,28 \%)$ and had received a PD1/PDL1 inhibitor monotherapy (31/36, 86\%) with $5 / 36(14 \%)$ combination therapy. Large joint involvement (hip/knee) was noted in 53\% (19/36), small joints of hand $25 \%$ (9/36), and spine 14\% (5/36). Two-thirds (24/36) suffered multiple joint involvement. Three of 36 (8\%) had CTCAE grade 3, 14 (39\%) grade 2 and 19 (53\%) grade 1 manifestations. Symptom onset ranged from six days to 33.8 months with median of 5.2 months after ICI initiation; 5 patients suffered ICI-aOA after ICI cessation (0.6, 3.5, 4.4, 7.3 and 15.4 months after ICI cessation) (figure 1). Most common form of therapy was intra-articular corticosteroid injections only $(15 / 36,42 \%)$ followed by NSAIDs only $(7 / 36$, $20 \%$ ) (figure 2). Twenty patients (56\%) experienced other irAEs, with rheumatic and dermatologic being the most common. All three patients with high-grade ICI-aOA also had another irAE diagnosis at some point after ICI initiation.

Conclusions ICI-aOA should be recognized as an adverse event of ICI immunotherapy. Early referral to a rheumatologist can facilitate the distinction between ICI induced inflammatory arthritis from post-ICI mechanical arthropathy, the latter of which can be managed with local therapy that will not compromise ICI efficacy.

Ethics Approval Collection of patient data was approved by local Institutional Review Boards at respective institutions: Hospital for Special Surgery in New York (HSS IRB \# 20171898), University of Chicago in Chicago, Illinois (IRB150837) and Austin Health in Melbourne, Victoria, Australia (HREC/ 18/Austin/102).

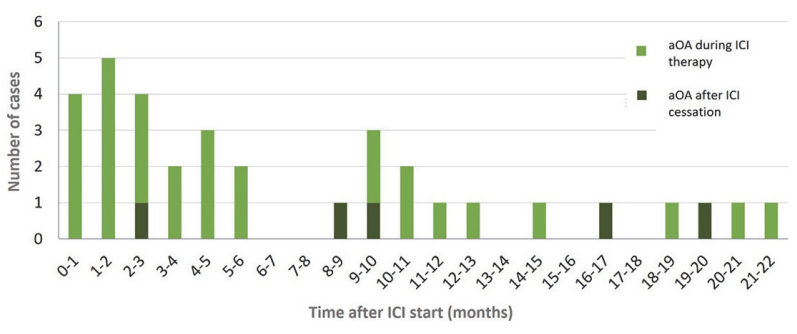

Abstract 817 Figure 1 Incidence of $\mathrm{ICl}-\mathrm{aOA}$ (activated osteoarthritis after immune-checkpoint inhibitor) ranged from the first month after $\mathrm{ICI}$ initiation up until month 22 after $\mathrm{ICl}$ initiation, with most cases occurring in the first 6 months after start of $\mathrm{ICl}$. Five of 36 patients experienced $\mathrm{ICl}-\mathrm{aOA}$ after $\mathrm{ICl}$ cessation $(0.6,3.5,4.4,7.3$ and 15.4 months after $\mathrm{ICl}$ cessation), corresponding to presentation after $\mathrm{ICl}$ initiation as follows: 2.0, 9.6, 19.1, 8.7 and 16.1 months after $\mathrm{ICl}$ initiation, respectively (as denoted in darker color). ICl: Immunecheckpoint inhibitor, NSAIDs: Non-steroidal anti-inflammatory drugs, DMARDs: Disease modifying anti-rheumatic drugs

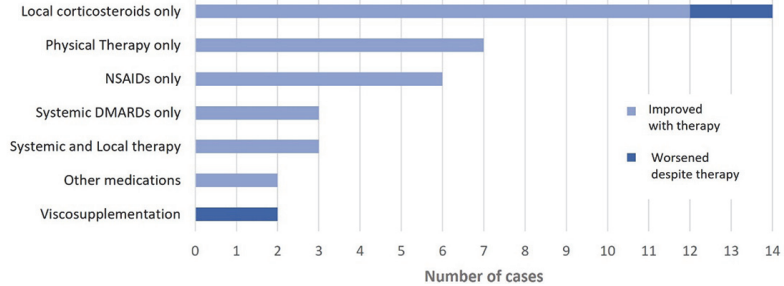

Abstract 817 Figure 2 Therapeutic option most used was local or intra-articular corticosteroid therapy, followed by conservative management with physical therapy only then NSAIDs. Most patients experienced improvement in signs and symptoms with treatment. ICl: Immune-checkpoint inhibitor, NSAIDs: Non-steroidal anti-inflammatory drugs, DMARDs: Disease modifying anti-rheumatic drugs

http://dx.doi.org/10.1136/jitc-2021-SITC2021.817 